Ophthalmologe $2019 \cdot 116: 28-32$

https://doi.org/10.1007/s00347-018-0821-y

(c) Springer Medizin Verlag GmbH, ein Teil von Springer Nature 2018

CrossMark
B. Seitz ${ }^{1}$ N. Graf ${ }^{2} \cdot$ M. Menger ${ }^{2}$ D. Monz ${ }^{2}$ B. Käsmann-Kellner ${ }^{1}$

${ }^{1}$ Klinik für Augenheilkunde, Universitätsklinikum des Saarlandes UKS, Homburg/Saar, Deutschland

${ }^{2}$ Medizinische Fakultät, Universität des Saarlandes UdS, Homburg/Saar, Deutschland

\section{Etablierung einer PJ-Faculty an der Medizinischen Fakultät der Universität des Saarlandes UdS}

\author{
"Docendo discimus", "Transparenz und \\ Kommunikation"
}

\section{Einleitung}

\section{Allgemeine Überlegungen zur Lehre im praktischen Jahr (PJ) des Medizinstudiums}

Universitäten sind Multifunktionseinrichtungen, in deren Mittelpunkt die Einheit von Forschung und Lehre und deren Gleichrangigkeit stehen. Bei einem Universitätsklinikum kommen als drittes Aufgabengebiet die Krankenversorgung sowie die Vorbereitung der Studierenden auf ihren Berufsalltag hinzu. Konfliktträchtige Situationen v. a. hinsichtlich der Ressource "Zeit" sind daher bei der medizinischen Lehre oft vorprogrammiert und waren auch bei unserem Wunsch, die Lehre im PJ zu verbessern, mitauslösende Gründe. „Gute Lehre“ rückt immer mehr in das Zentrum hochschulpolitischer wie universitärer Bemühungen. Bei näherer Betrachtung der Literatur zu Schlagwörtern wie „Qualitätssicherung in der Lehre" wird deutlich, dass es eine wachsende Vielschichtigkeit der Indikatoren für "gute Lehre" gibt und dies die Beurteilung der Lehre erschwert [1].

Nicht erst seit der Eröffnung der Möglichkeit im Jahr 2016, das PJ an jedem Universitätsklinikum und Lehrkrankenhaus außerhalb des Universitätsklinikums des Saarlandes UKS und seiner Lehrkrankenhäuser ableisten zu können, nahm die Anzahl der PJ-Studierenden Jahr für Jahr ab. Gerade bei der in einem Uniklinikum knapp be- messenen Ressource Zeit wollten wir dezidiert die Verpflichtung jedes Arztes im UKS zur Lehre positiv hervorheben [2] und einen 10-Punkte-Plan zur Prioritätensetzung entwickeln, um sowohl zu den Nachbaruniversitäten als auch zu den nichtuniversitären Nachbarkliniken nachhaltig das Profil der PJ-Lehre zu schärfen.

Evaluation ist ein unabdingbares Instrument der Qualitätssicherung. Wir entschlossen uns daher, eine institutionell verankerte und stark individualisierte Lehrevaluationskultur (Gestaltungsevaluation) zu etablieren, insbesondere weil die Zufriedenheit der Studierenden bezüglich der PJ-Lehre in verschiedenen Kliniken zum Teil deutlich schwankte. Der "Augenblock“ nach Ulmer Vorbild wird von den Studierenden seit 10 Jahren in Homburg sehr gut bewertet [3]. In anderen Fachbereichen war jedoch zum Teil eine deutliche Diskrepanz zwischen der Fremdeinschätzung durch die Studierenden und der Selbsteinschätzung der Lehrstuhlinhaber und ihrer Lehrbeauftragten zur Qualität ihrer Lehre festzustellen. Im Sinne des allgemeinen universitären Bestrebens, innovative Lehr-Lern-Konzepte voranzutreiben, beschlossen wir die Restrukturierung der PJ-Lehre.

\section{Vorstellung Zehn-Punkte-Pro- gramm an Sofortmaßnahmen zur Verbesserung der PJ-Lehre an der UdS}

Im Dezember 2015 wurde in der letzten Sitzung des Erweiterten Fakultätsrates EFR des Jahres die Task Force PJLehre unter der Federführung des PJ-Beauftragten der Fakultät, Herrn Prof. Dr. Berthold Seitz, Direktor der UniversitätsAugenklinik des Saarlandes, und des Studiendekans der Fakultät, Herrn Prof. Dr. Norbert Graf, etabliert. Folgende Mitglieder wurden in diese Task Force berufen: Die Professoren Bals (Pneumologie), Glanemann (Allgemeinchirurgie), Lammert (Gastroenterologie), Menger (Experimentelle Chirurgie), Schick (HNO) und Volk (Anästhesie), die Studierenden Frau Asen, Frau Kerner, Frau Schmid und Frau Turan sowie Herr Dr. Monz als Repräsentant des Studiendekanats, der eine unverzichtbare Rolle bei Terminierung, Protokollierung und Organisation der Task Force einnahm und bis heute bei der PJ-Faculty einnimmt.

Nach 3 zeitnahen Sitzungen der Task Force PJ-Lehre unter expliziter Einforderung von studentischem Input wurde von Herrn Seitz das folgende 10-PunkteSofortprogramm zur Verbesserung der PJ-Lehre an der UdS vorgestellt und in der Fakultät mit großer Mehrheit verabschiedet. Seitdem unterliegt dieses Konzept einem kontinuierlichen Verbes- 


\section{Infobox 1 Informationen zum praktischen Jahr auf der Webseite des}

UKS (http://www.uniklinikum-saarland.de/de/lehre/humanmedizin/ praktisches_jahr/)

Folgende Flyer, Merkblätter und Checklisten sind auf der Webseite zu finden:

- PJ-Flyer aktuell http://www.uniklinikum-saarland.de/fileadmin/UKS/Lehre/Studiengaenge/ Humanmedizin/Praktisches_Jahr/PJ-Flyer.pdf

- PJ-Broschüre aktuell http://www.uniklinikum-saarland.de/fileadmin/UKS/Lehre/ Studiengaenge/Humanmedizin/Praktisches_Jahr/PJ-Broschuere.pdf

- PJ-Logbuch (http://www.uniklinikum-saarland.de/de/lehre/humanmedizin/praktisches_jahr/ pj_logbuecher/)

- PJ-Curriculum (http://www.uniklinikum-saarland.de/de/lehre/humanmedizin/praktisches_ jahr/curricula/)

- Merkblatt für PJ-Studenten: http://www.uniklinikum-saarland.de/fileadmin/UKS/Lehre/ Studiengaenge/Humanmedizin/Praktisches_Jahr/Merkblatt_fuer_PJ_27.06.2016.pdf

- Geschäftsordnung der PJ-Faculty http://www.uniklinikum-saarland.de/fileadmin/UKS/Lehre/ Studiengaenge/Humanmedizin/Praktisches_Jahr/20170503_Geschaeftsordnung_PJ_Faculty_ Homburg.pdf

- Antragsformular für PJ-Lehr-LOM seit 2017 http://www.uniklinikum-saarland.de/fileadmin/ UKS/Lehre/Studiengaenge/Humanmedizin/Praktisches_Jahr/Antrag_LOM_fuer_PJ.pdf

- Checkliste PJ-Peer Review http://www.uniklinikum-saarland.de/fileadmin/UKS/Lehre/ Studiengaenge/Humanmedizin/Praktisches_Jahr/Checkliste_Peer_review.pdf

serungsprozess in der neu gegründeten PJFaculty (s. unten).

\section{Grundlegende organisatorische Dinge müssen immer und überall funktionieren}

Am ersten Tag des PJ-Tertials treffen sich alle neuen PJ-Studierenden mit Herrn Seitz, dem Studiendekan, Herrn Graf, und Frau Orlich vom Studiendekanat um 8:00 Uhr im Hörsaal der Augenklinik, um nach einer kurzen organisatorischen Einführung ihr Namensschild, die Evaluationsbögen und eine Liste mit allen PJBeauftragten, Direktoren und zuständigen Sekretärinnen (mit Telefon, Fax und E-Mail-Adresse) in Empfang zu nehmen. $\mathrm{Zu}$ diesem Treffen sind auch alle PJ-Beauftragten der Kliniken eingeladen, die nach der Einführung „ihre“ PJ-Studierenden mit in die Kliniken nehmen. $\mathrm{Zu}$ diesem Zweck war es unerlässlich, dass neben den allgemeinen Lehrbeauftragten feste PJ-Beauftragte und ein kompetentes und erreichbares Studierendensekretariat in jeder Klinik benannt wurden. In der Klinik bekommt jeder PJ-Studierende nötige Schlüssel, Kittel und Computerzugang für SAP und ggf. eine fachspezifische elektronische Patientenakte (z.B. FIDUS in der Augenklinik [4]).

Mittlerweile wurde vom Vorstand des UKS unter Mitwirkung des neuen Personaldezernenten Herrn Christian Mül- ler auf Antrag der PJ-Faculty beschlossen, dass die PJ-Studierenden zum Ausdruck der Wertschätzung einen Ausweis wie Ärzte bekommen.

\section{Die Online-Evaluationsbögen sind klinikbezogen und dozentenbezogen}

Die Evaluation ist ein sehr wesentlicher Bestandteil der Selbstkontrolle von Lehrleistungen und studentischer Akzeptanz. Eine ernst zu nehmende Evaluation hat kein Ranking im Sinn, sondern das Ziel, den Dozenten Rückmeldung im Sinne von Überprüfung der angestrebten Ziele zu geben. Deshalb muss sie nicht nur klinikbezogen sondern auch dozentenbezogen sein. Besonders in den großen Fächern (innere Medizin mit 5 Kliniken, Chirurgie mit 4 Kliniken) muss klar nachvollziehbar sein, welcher Lehrer wann wie evaluiert wird. Die Studierendensekretariate erinnern die PJ-Studenten an die Evaluation, wenn die PJ-Scheine abgeholt werden („Geben und Nehmen").
3. Werbung für das PJ sollte schon frühzeitig durch gute studentische Lehre in Vorklinik und Klinik sowie eine authentische Willkommenskultur beginnen

Im Blockpraktikum war oft zu wenig Zeit für die Studierenden, und diese fühlen sich nicht gut aufgenommen. Das Blockpraktikum als "Vorstufe“ zum PJ wurde verbessert und attraktiver gemacht. Gute Lehre, die neben dem eigentlichen Curriculum in Wahlfächern angeboten wurde, ist ins Blockpraktikum integriert worden. Gerade in den großen Fächern Innere und Chirurgie musste das Blockpraktikum deutlich inhaltlich und v. a. organisatorisch verbessert werden. Dafür erhielt das Blockpraktikum mehr Struktur und ein Curriculum, sodass die Studenten nicht nur ,mitlaufen“, sondern aktiv und kompetent unterrichtet werden. Auch bei den Ausbildern in der Klinik wurde mehr Bewusstsein für die Bedeutung des Blockpraktikums und die Wertigkeit der Studierenden als zukünftige Kolleginnen und Kollegen in Zeiten des Fachkräftemangels geschaffen.

Ein wesentliches Projekt zur Werbung von Studierenden in Homburg und auch außerhalb Homburgs stellen PJ-Flyer und PJ-Broschüre (• Infobox 1) dar. Die Broschüre fokussiert auf Homburg als attraktiven PJ-Standort sowie die 20 Kernkompetenzen des PJs in Homburg, die JEDER PJ-Studierende sicher vermittelt bekommt, sowie die "Sahnehäubchen", die jede individuelle Klinik im Universitätsklinikum des Saarlandes als universitäres Krankenhaus der Maximalversorgung den PJ-Studierenden zu bieten hat.

Folgende Adressaten für PJ-Broschüre und -Flyer wurden zusammen mit den Studierenden festgelegt:

a. Fachschaften anderer Universitäten mit Studiengang Medizin (ca. 35 in Deutschland), je 20 Stück, Adressen der Fachschaften über unsere Fachschaft, Verpackung und Versand via Studiendekanat,

b. Internetseite der Fachschaft Medizin Homburg und des Studiendekanats (immer aktuellste Version),

c. Hörsäle,

d. Bibliothek, 
e. Mensa,

f. Institut für Allgemeinmedizin (Professur Dr. Jäger),

g. Centre Hospitalier Luxembourg und Emile Mayrisch Esch.

Zusätzlich werden diese Broschüren und Flyer bei der Einführung in die klinische Medizin (Einführungsveranstaltung im 1. klinischen Semester) bereits ausgeteilt.

\section{Frühzeitige Informations- veranstaltung zum PJ}

Die Task Force PJ-Lehre hielt es für sehr wichtig, dass die Studierenden in Homburg möglichst früh darüber informiert werden, was genau sie in Homburg im PJ erwartet, was ihnen geboten wird und welche Vorteile Homburg bietet. Diese erfolgreiche interne PJ-Informationsveranstaltung wird seit 2016 für alle Studierenden im klinischen Studienabschnitt regelmäßig einmal im WS angeboten. Es stellen sich alle 5 Kliniken für innere Medizin und alle 4 Kliniken für Chirurgie sowie möglichst viele Wahlfächer vor. Pro Fach sollten die Chefs, die PJ-Lehrbeauftragten sowie 1 bis 2 aktuelle PJ-Studierende anwesend sein. Für allgemeine Fragen zum PJ stehen zusätzlich Frau Orlich vom Studiendekanat und Herr Müller vom Landesprüfungsamt zur Verfügung.

\section{Logbücher und individuelle PJ-Curricula müssen existent und bekannt sein und von Lehrenden und PJ-Studierenden umgesetzt werden}

Die Inhalte der PJ-Website auf der Homepage des UKS wurden aktualisiert und inhaltlich und organisatorisch besser strukturiert: wenige Punkte, die wirklich wichtig sind (•Infobox 1). Für jede Klinik ist unmittelbar das PJ-Logbuch und PJCurriculum ersichtlich (•Infobox 1). Das allgemeine PJ-Curriculum wurde überarbeitet und aktualisiert. Auf der Homepage der einzelnen Kliniken werden jetzt unter „Lehre PJ“ auch die PJ-Lehrbeauftragten mit Telefonnummer und E-MailAdresse genannt.

Es wurden über alle Fächer hinweg 20 Kernkompetenzen definiert, die den
PJ-Studierenden klinikübergreifend vermittelt werden sollen, sodass am Ende des PJs alle Studierenden diese Fähigkeiten erlangt haben (vgl. PJ-Flyer und -Broschüre). Jeder PJ-Studierende sollte ein Patientenzimmer zugewiesen bekommen, sodass z. B. Kompetenzen wie Patientenvorstellung beim Oberarzt oder Chef und das Schreiben von Arzt- und Entlassungsbriefen erlernt werden.

\section{Persönliche Gespräche zu Beginn und Ende des Tertials}

Im Rahmen der Willkommenskultur findet ein individuelles persönliches $\mathrm{Ge}$ spräch zwischen dem PJ-Studierenden und dem Chef zu Beginn (Ziele festlegen) und nach dem dritten Monat des Tertials („Reflexion“, was noch fehlt ...) statt.

\section{Sham-Prüfung als Vorbereitung auf das Examen}

Sham-Prüfungen werden den PJ-Studierenden rechtzeitig im PJ als Chance angeboten, dürfen jedoch keineswegs zur Pflicht gemacht werden. Die Prüfungen werden durch den Chef oder einen motivierten Oberarzt, der im Staatsexamen selbst prüft, durchgeführt. Sham-Prüfungen sind so gestaltet, dass man den Prüfling zunächst ein Organ untersuchen lässt. Die Prüfung orientiert sich an OSCE-Prüfungen („objective structured clinical examination“) und enthält 4 bis 5 definierte Untersuchungsschritte, die der Prüfling auf jeden Fall erfüllen sollte. Untersuchungsschritte, die für eine bestimmte Untersuchung als wichtig angesehen werden, werden auch während der Ausbildung transparent gemacht und vermittelt. Neben den Sham-Prüfungen wurden die Prüfergruppen für das letzte Staatsexamen besser definiert und vermehrt M3-Prüfer ins PJ involviert.

\section{Strukturelle Verbesserung der Lehre im PJ}

\subsection{Homburger PJ-Faculty}

Die PJ-Lehre/Ausbildung wurde stärker personalisiert. Neben den Lehrbeauftragten sind heute den Studierenden weitere kompetente Ansprechpartner für das PJ in den Kliniken bekannt. Eine Geschäftsordnung der „Homburger PJFaculty“ wurde etabliert am 03.02.2017 (- Infobox 1). Ihr gehören u.a. der Dekan, Studiendekan und PJ-Beauftragte der Fakultät, 4 Studierende, alle Klinikdirektoren und insbesondere alle PJLehrbeauftragte an. Sie trifft sich regelmäßig alle 3 Monate. Die Aufgabe der PJ-Faculty ist der aktive kontinuierliche Verbesserungsprozess (Organisation und inhaltliche Optimierung) der PJ-Lehre an der UdS in Homburg. Sie bestimmt z. B. über die Vergabe des PJ-Lehrpreises (s. unten: 8.3.) und der Lehr-LOM-PJ (leistungsorientierte Mittelvergabe für das PJ) nach den Regeln der Fakultätsleitung.

\subsection{Die Lehr-LOM-Mittel werden um $50.000 €$ zur Unterstützung guter Lehre im PJ erhöht}

Finanziell wird die verbesserte PJ-Lehre nach Beschluss der Fakultät durch die Lehr-LOM unterstützt. Die Vergabe erfolgt auf Antrag (- Infobox 1) bis zum 30. Juni jeden Jahres und nach Begutachtung durch die PJ-Faculty einmal im Jahr. Der Antragsteller hat auf folgende Fragen überzeugende Antworten zu präsentieren: (1) durchschnittliche Anzahl der PJStudierenden/Tertial während der letzten 3 Jahre, (2) Ergebnis bisheriger Evaluationen, (3) Gegenstand und Konzeption des $\mathrm{zu}$ fördernden Vorhabens, (4) Bestandaufnahme und Mängelanalyse (ggf. unter Verweis auf Umfragen, Evaluationen), (5) Ziele des Vorhabens und Beitrag zur Verbesserung des PJ, (6) Methoden und Instrumente zur Qualitätskontrolle mit Kriterien zur Bewertung der Ergebnisse nach Abschluss des Vorhabens, (7) Kostenaufstellung mit Begründung, (8) Personalkosten, (9) Sachkosten, (10) Folgekosten/Wartungskosten, (11) langfristige Planung mit Begründung, wie das Projekt auch langfristig ohne weitere Gelder in die PJ-Lehre integriert werden kann.

Folgende Projekte wurden im Jahr 2017 gefördert:

1. Tutdibi, Erol; Nourkami-Tutdibi, Nasenien (Pädiatrie): Strukturierte Ultraschall-Ausbildung des Abdomens und Herzens von Studierenden für Studierende im PJ Genehmigt: 7250,00€ 
2. List, Franka; Armbruster, Werner (Anästhesiologie): Hands-On-Training im Anästhesie-PJ - 1 Tag im Simulatorzentrum Genehmigt: $6000,00 €$

3. Jäger, Johannes (Allgemeinmedizin): Peer-teaching and Peer-monitoring of PJ-students in General Medicine Genehmigt: 3001,92€

4. Scherber, Philipp; Glanemann, Matthias (Allgemeine Chirurgie): Surgical Skills Lab - Trainingslabor für laparoskopisches Operieren und chirurgische Basistechniken Genehmigt: 7500,00€

5. Volk, Thomas; Groesdonk, Heinrich; Hüppe, Tobias (Anästhesiologie): Point-of-Care Ultraschall in der Akut- und Notfallmedizin Genehmigt: $8000,00 €$

\subsection{PJ-Lehrpreis}

Gute Lehre im PJ durch Assistenzärzte/-innen oder junge Fachärzte/-innen wird im UKS Newsletter, per UKS Rundmail und in der Presse bekannt gemacht und durch einen PJ-Lehrpreis in Höhe von $4000 €$ belohnt. Der Preis wird auf Vorschlag der Studierenden (Deadline 30.10. des Jahres) nach Aussprache im Rahmen der PJ-Faculty vergeben (Voraussetzungen: 1. Begründung des Vorschlagenden, 2. Lehrkonzept des Lehrenden, 3. kurzes CV des Lehrenden). Der Preis wird einmal im Jahr während der Examensfeier im Dezember vergeben. Um eine gebührende Signalwirkung zu haben, werden Preisträger auch auf der Website des Studiendekanats, in der überregionalen Presse, im UdS-Präsidium und den Ministerien bekannt gemacht und damit „estimiert“. Der erste PJ-Lehrpreis des „Fördervereins für Augenheilkunde am UKS“ wurde am 15.12.2017 im Rahmen der Examensfeier von Herrn Seitz an Herrn Dr. Tobias Hüppe (Anästhesiologie) verliehen. Derzeit finanziert die Willy Robert Pitzer-Stiftung in Frankfurt den jährlichen PJ-Lehrpreis.

\section{Peer-Review der PJ-Ausbildung}

Der Chef und der PJ-Beauftragte einer Klinik überprüfen als Fachfremde die PJ-Ausbildung einer anderen Klinik zusammen mit deren Chef und PJ-Beauf- tragten kritisch. Dies geschah zunächst zwischen „befreundeten“ Kliniken (z.B. Augenklinik und Gastroenterologie, Pädiatrie und Augenheilkunde), die ihre PJ-Ausbildung evaluieren und dadurch verbessern wollen. Es wurde ein Katalog an Kriterien entwickelt, die als wichtig für die PJ-Ausbildung angesehen werden, wie das jeweilige Curriculum und Logbuch, durchgeführte Fortbildungen und Gespräche zur Evaluation mit den aktuellen PJ-Studierenden (•Infobox 1).

\section{PJ-Aufwandsentschädigung}

Die in ihrem Studium geleistete Arbeit der PJ-Studierenden am Patienten und bei der Dokumentation stellt einen wesentlichen Beitrag zur Patientenversorgung - insbesondere in Zeiten des Fachkräftemangels - dar. Früher wurde den PJ-Studierenden in Homburg - im Gegensatz zu weiten Teilen der übrigen Nation - keinerlei Aufwandsentschädigung gezahlt. Seit dem 01.10.2016 wird - auf intensives Betreiben der Task Force PJLehre und des Ärztlichen Direktors Prof. Dr. B. Schick - für das erste Tertial am UKS 160,- €/Monat, für das 2 . Tertial am UKS 240,- €/Monat und für das dritte Tertial am UKS 400,- €/Monat vom UKSVorstand an die PJ-Studierenden bezahlt. Der aktuelle Stand des Aufwandsentschädigung steht im Merkblatt für PJ-Studenten, das man unter „Aufwandsentschädigung" findet (-Infobox 1). Das angestrebte Ziel sind 500,- $€$ pro Monat ab dem ersten Tertial.

\section{Schlussfolgerungen}

Originalität und Übertragbarkeit dieses Konzeptes zur Verbesserung der PJ-Lehre auf andere Standorte liegen auf der Hand. Die Einrichtung der „Task Force PJ-Lehre" hat zu einem Motivationsschub für gute Lehre im PJ an unserer Fakultät geführt und garantiert deren Nachhaltigkeit. Entscheidend für die Früchte der Maßnahmen sind aber auch die Transparenz der individuellen Umsetzung und die Kommunikation an die Kolleginnen und PJ-Studierenden durch eine umfassende individuelle Evaluation der einzelnen Kliniken und Lehrer.

\section{Korrespondenzadresse}

Prof. Dr. B. Seitz, ML, FEBO

Klinik für Augenheilkunde, Universitätsklinikum des Saarlandes UKS

Kirrbergerstr. 100, 66424 Homburg/Saar,

Deutschland

berthold.seitz@uks.eu

Interessenkonflikt. B. Seitz, N. Graf, M. Menger, D. Monz und B. Käsmann-Kellner geben an, dass kein Interessenkonflikt besteht.

\section{Literatur}

1. Geerling G, Seitz B (2015) Der Lehre verpflichtet (Editorial). Ophthalmologe 112:467

2. Goebels S, Viestenz AN, Seitz B (2017) Facharztweiterbildung an der Augenklinik des Universitätsklinikums des Saarlandes - Das Homburger Curriculum als Modell für die Facharztweiterbildung zum Augenarzt am Universitätsklinikum des Saarlandes (UKS). Ophthalmologe 114:930-994

3. Käsmann-Kellner B, Seitz B (2015) Studentische Lehre an der Augenklinik des Universitätsklinikums des Saarlandes (UKS) - Der Homburger AUGENBLOCK. Ophthalmologe 112:477-487

4. Spira-Eppig C, Eppig T, Bischof M, SchießIG, Milioti G, Käsmann-Kellner B, Carstensen H, Schick B, Seitz B (2018) Per aspera ad astra: 1 Jahr elektronische Patientenakte in Homburg/Saar - Erfahrungen mit "FIDUS" in der Klinik für Augenheilkunde am Universitätstklinikum des Saarlandes UKS. Ophthalmologe 115:868-877 
Hier steht eine Anzeige.

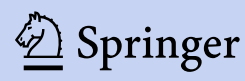

